\title{
A hierarchical approach for computing spin glass ground states
}

\author{
Jérôme Houdayer ${ }^{(1)}$ and Olivier C. Martin ${ }^{(2)}$ \\ 1) Institut für Physik, \\ Mainz, Germany \\ 2) Laboratoire de Physique Théorique et Modèles Statistiques, \\ bât. 100, Université Paris-Sud, F-91405 Orsay, France
}

(November 1, 2018)

\begin{abstract}
We describe a numerical algorithm for computing spin glass ground states with a high level of reliability. The method uses a population based search and applies optimization on multiple scales. Benchmarks are given leading to estimates of the performance on large lattices.

PACS Numbers : 75.10.Nr Spin-glass and other random models 75.40.Mg Numerical simulation studies

\section{INTRODUCTION}

\section{GRAPHS AND HAMILTONIANS}

Discrete optimization plays a central role in many engineering problems such as scheduling and electronic circuit design, but it is also important in fundamental science. One major open problem there concerns the nature of the energy landscape of optimization problems with quenched disorder. It is generally agreed that these energy landscapes are rugged, but are they self-simplar as predicted by the scaling theories of spin glassesule? Despite many years of controversy, this issue is still unsettled. In order to make progress, it is useful to be able to compute the ground states of these disordered systems 3 . Indeed, by applying sensitivity analysis, that is by considering how the ground state changes when the parameters specifying the optimization problem vary, one can probe the energy landscape and measure its scaling exponents. For some types of optimization problems, finding ground states can be achieved with algorithms whose CPU time grows polynomially with the problem size (the size is the number of discrete variables in the problem). However, in many other interesting cases, finding the ground state is an NP-hard problem so efficient algorithms are particularly called for. In this work, we present a method for computing ground states heuristically; even though our algorithm is not guaranteed to provide the optimum as its output, the ground states can be found for significantly larger systems than with methods having such a guarantee. For instance the ground state of a $10 \times 10 \times 10$ Edwards-Anderson spin glass can be computed in a few minutes on a personal computer, while $13 \times 13 \times 13$ lattices can be solved on larger computers. Results using exact methods such as branch and bound have been published $\$$ but only for much smaller sizes $(4 \times 4 \times 4)$.

In the next section, we give the general framework in which we work. Section III introduces the main features of our algorithm which embeds both a local search and renormalization into a genetic algorithm; then section IV describes the over-all algorithm architecture. Finally, in section $\mathrm{V}$, we explain how the algorithm behaves in practice on benchmark problems.
We consider an Ising spin glass, defined on an arbitrary non-oriented graph $G$; the Hamiltonian or energy function we seek to minimize is

$$
H=-\sum_{\{i, j\} \in E} J_{i j} S_{i} S_{j}-\sum_{i \in V} h_{i} S_{i} .
$$

The "spins" $S_{i}$ are of the Ising type, $S_{i}= \pm 1$, and lie on the vertices or "sites" of $G$. $N$ is the size of our system, that is the number of these vertices. $E$ is the set of edges of $G$; each edge connects two vertices. Finally, the $J_{i j}$ and the $h_{i}$ can be any real numbers; the $J_{i j}$ lie on the edges of $G$ while the $h_{i}$ lie on its vertices. From here on, we call an instance the specification of all the parameters of the Hamiltonian, that is the specification of the graph $G$ and of all the parameters $J_{i j}$ and $h_{i}$. Similarly, we call a (spin) configuration the assignment of the values of the $S_{i}$ (for all $\left.i, 1 \leq i \leq N\right)$.

The Hamiltonian in equation 11 can be used to represent an arbitrary spin glass, be it on a lattice like the Edwards-Anderson (EA) modelE, or on a random graph as for diluted mean field spin glasses. It can also be used to represent a random field Ising model (RFIM) with or without disorder in the bond strengths. The algorithm we present in this work does not take advantage of any structure in $G$ or in the parameters defining the Hamiltonian; because of this, it cannot be expected to be competitive in the special cases where finding the ground state is a polynomial problem. (Note that 2-dimensional spin glasses and the RFIM fall into this class.) Nevertheless, in the other cases we have found the algorithm to be very effective. It is possible that improvements could be realized by taking advantage of additional structure in $H$, but we shall not investigate that issue here.

\section{COMPONENTS OF THE ALGORITHM}

The algorithm we present is a genetic algorithm: we evolve a population of configurations from one generation to the next. The key elements of our approach are: (i) 
the use of an embedded local search; (ii) the incorporation of a renormalization procedure among parents in the population which allows one to consider multiple length scales; (iii) the use of recursion. Because of these features, we talk of a "Genetic Renormalization Algorithm" (GRA).

A previous GRA algorithm was described in reference $\mathrm{\theta}$. For the readers aware of that work, our new approach has a different structure for the recursive calls, leading to an exponential speed-up in $N$ compared to the older method. Before describing our new algorithm, we first go over its main components.

\section{A. Local search}

Let us first define a few terms. Consider a configuration and one of its spins $S_{i}$. We call gain of that spin the decrease in the configuration's energy when $S_{i}$ is flipped. (The gain is thus minus the change in the total energy.) A spin is said stable (resp. unstable) if its gain is negative (resp. positive). Spin $S_{i}$ is said to be more unstable than $\operatorname{spin} S_{j}$ if $\operatorname{gain}\left(S_{i}\right)>\operatorname{gain}\left(S_{j}\right)$.

It is straightforward to extend these single spin definitions to sets of spins. We shall focus on the case of clusters; a cluster is a set of spins (vertices) which is connected (using the edges of $G$ ). Then, extending the previous definitions, the gain of a cluster is just minus the change of the total energy when all the spins of the cluster are flipped. Similarly, a cluster can be stable or unstable, etc...

A local search is a procedure that attempts to lower the energy of a configuration by repeatedly changing a few variables at a time, accepting only improving changes. For our system, this amounts to flipping sets of spins with positive gain until no more favorable sets are found. Local search covers many different methods because there is much freedom in the way one searches for and selects these sets. The method we have developped for our GRA is inspired from the Kernighan-Lind algorithm. In that class of local search algorithms, the number of variables that is changed at a time is not set before-hand and in practice can be quite large. For our local search, we force the set of spins that will be flipped to be connected, that is we restrict ourselves to clusters. Indeed, if there is a set of spins with positive gain, at least one connected component of that set has a positive gain.

Our search for a "good" cluster proceeds as follows. First we choose a starting spin; it defines the initial cluster. Second, we successively add new and promising spins to the current cluster, maintaining the connectivity property. During this growth process, the gain of the cluster can go positive or negative, up or down. Third, we stop growing the cluster when things no longer look promising. Finally, we consider all the gains generated during the cluster's growth and select the largest one. If that gain is strictly positive, we flip the corresponding clus- ter, generating an improved configuration. These steps correspond to one pass of the local search. We perform multiple passes until the search for an improving cluster fails; then a local minimum of $H$ has been reached and the local search is finished.

Naturally, the description we have given of our local search is rather schematic; one has to implement in the code how to choose the starting spin, what is a promising spin, etc... The reader interested in these details will find them in Appendix 1. Other types of local searches could be used instead; our choice is motivated by a trade-off between speed and quality.

\section{B. Renormalization}

Given a spin glass Hamiltonian for $N$ spins and at least two spin configurations, there is a natural definition of a block spin; from that, one can extract an exact renormalized spin glass Hamiltonian associated with a system having in general $N^{\prime}<N$ spins. Such a renormalization procedure was first proposed by Kawashima and Suzuki to keep this paper self-contained, we describe this method again.

Let equation 1 be the Hamiltonian of the system and $G$ be the associated graph. Suppose we have $k$ spin configurations $\left\{S_{i}^{(1)}\right\},\left\{S_{i}^{(2)}\right\}, \ldots,\left\{S_{i}^{(k)}\right\}$. We then define the signature at site $i$ to be the following vector of \pm 1 values:

$$
\vec{\sigma}_{i}=\left(S_{i}^{(1)} S_{i}^{(2)}, S_{i}^{(1)} S_{i}^{(3)}, \ldots, S_{i}^{(1)} S_{i}^{(k)}\right) .
$$

Two sites $i$ and $j$ have the same signature if and only if the spins at those sites have the same relative orientation (parallel or anti-parallel) in all of the $k$ configurations. Now we partition the sites of $G$ according to their signature. Furthermore, given a set of sites of identical signature, we further subdivide this set into clusters (i.e., the desired subsets are the connected components of that set, where as usual connectivity is defined using the edges of $G)$. Thus to each site $i$ corresponds a maximal connected cluster $A(i)$ of sites; then for any site $j$,

$$
A(j)=A(i) \Rightarrow \vec{\sigma}_{j}=\vec{\sigma}_{i} .
$$

For each cluster $A$, we introduce the "block-spin" $S_{A}$ to be +1 if all the spins in $A$ are parallel to those of the first configuration, and -1 if they are all anti-parallel. Thus for configuration 1 we have $S_{A}=1$ for all $A$ while for the $n$th configuration $(1 \leq n \leq k)$ we have

$$
S_{A}^{(n)}=S_{i}^{(1)} S_{i}^{(n)} \text {, for any } i \in A .
$$

as can be seen from equations 2 and 3 .

The exact Hamiltonian for these block spins is of the form

$$
H^{\prime}\left[\left\{S_{A}\right\}\right]=-\sum_{(A, B) \in E^{\prime}} J_{A B} S_{A} S_{B}-\sum_{A \in V^{\prime}} h_{A} S_{A},
$$




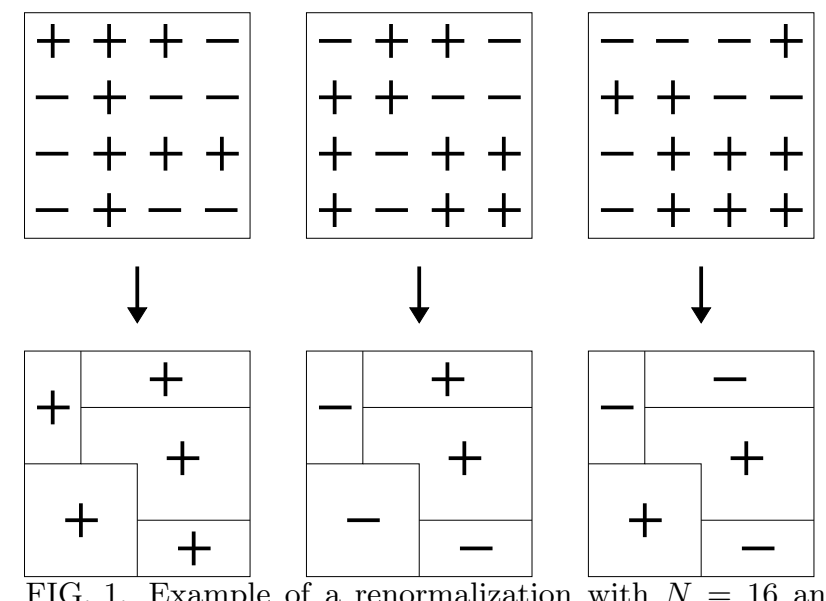

FIG. 1. Example of a renormalization with $N=16$ and $k=3$ for nearest neighbor interactions. If the interactions had been long range, the top left and the bottom right clusters would be merged since they would form a connected set of sites with the same signature.

where $V^{\prime}$ is the set of the $A$ clusters and $E^{\prime}$ is the set of edges of the corresponding "renormalized" graph $G^{\prime}$. Note that two blocked spins $S_{A}$ and $S_{B}$ are neighbors in $G^{\prime}$ if at least one spin of the cluster $A$ is a neighbor (according to $G$ ) of at least one spin of the cluster $B$. A simple computation gives the couplings between the block spins:

$$
J_{A B}=\sum_{i \in A} \sum_{j \in B} J_{i j} S_{i}^{(1)} S_{j}^{(1)} .
$$

Similarly, the fields are:

$$
h_{A}=\sum_{i \in A} h_{i} S_{i}^{(1)} .
$$

Thus we have

$$
H\left[S_{i}^{\alpha}\right]=H^{\prime}\left[S_{A}^{\alpha}\right]+H_{c},
$$

where $H_{c}$ is a constant representing the interaction energy inside the clusters

$$
H_{c}=-\sum_{A \in V^{\prime}} \sum_{i, j \in A} J_{i j} S_{i}^{(1)} S_{j}^{(1)} .
$$

In figure 1 we give an illustration of the construction of the clusters (block spins) generated by the renormalization when using 3 configurations. (In this example, $G$ is a $4 \times 4$ lattice.)

The renormalization procedure can be reversed in the sense that if you have a spin configuration for $H^{\prime}$ you can recover the corresponding one for $H$ using equation 1 (provided you remember $S_{i}^{(1)}$ and the definition of the clusters). We will call this operation the raising of a configuration.

The idea is then as follows. If you have a set of configurations for $H$, you first use the renormalization procedure to produce configurations $\left\{S_{A}^{(n)}\right\}$ associated with a smaller number of spins (these are the block spins). Second, you improve these configurations by a suitable optimization procedure. Finally, you raise these improved configurations, obtaining new configurations for the initial Hamiltonian but with lower energies than previously.

\section{Population evolution}

The main problem encountered when searching for the ground state via local search is that asymptotically one has a fixed percentage error on the (extensive) ground state energy and so the probability of reaching the ground state goes to zero exponentially with $N$. To postpone this bad behavior one could improve the local search but that is computationally costly. Furthermore, in spin glasses (as in most difficult optimization problems), low energy configurations may differ from the ground state by a "large" number of spins, in fact a number growing linearly with $N$. (This is expected to happen when the overlap probability distribution $P(q)$ is broad, signaling replica symmetry breaking 9 ). When this happens, improvements in the local search algorithm are doomed to be ineffective. So instead we appeal to renormalization in order to optimize on larger length scales while still using the same local search! As an extra bonus, one may be able to perform optimization on all scales if the renormalization is done recursively...

Using the local search and the renormalization together requires working with a population of configurations. Our method is thus a generalized genetic algorithm; we evolve a population of configurations whose energies we try to minimize. To do this, we repeatedly choose subsets of the population (the parents) to which we apply the renormalization procedure. For each such subset, we then obtain a smaller spin glass instance and a corresponding set of configurations. We optimize (see next section) these configurations and deduce new configurations for the original Hamiltonian (the children) that have a lower energy than their parents. When all the parents have produced enough children, the parents are replaced by the children, giving rise to a new generation. When no further improvements are possible, the algorithm stops.

One of the essential features of our approach is that we impose the children to be better than their parents. Let us define this notion mathematically as follows. We say that a population $P^{\prime}$ is more optimized than a population $P$ if there is a mapping $f$ from $P$ to $P^{\prime}$ such that: (i) $f$ is onto, i.e., the mapping of $P$ covers all of $P^{\prime}$; (ii) $f(C)=C^{\prime} \Rightarrow H\left(C^{\prime}\right) \leq H(C)$. Furthermore, we say that $P^{\prime}$ is strictly more optimized than $P$ if $f$ is not one-to-one or if $H(C)>H(f(C))$ for at least one $C$. These definitions introduce a partial ordering relation on populations. According to this relation, you obtain a strictly more optimized population in the following cases: (i) you remove the worst configuration (greatest 
energy); (ii) you remove duplicated configurations; (iii) you improve at least one configuration; (iv) you replace a sub-population by a strictly more optimized one. In our algorithm, duplicated configurations are removed, so all the configurations are different in any given population.

\section{ARCHITECTURE OF THE ALGORITHM}

\section{A. Recursion: the Next_Generation function}

We can now discuss more precisely how the algorithm works and in particular how the children are produced using a recursive call to the renormalization procedure. The heart of this is a function that we call "Next_Generation". We first describe its main ingredients; details will be added later in Appendix 2. For its input, this function takes an instance (i.e., a Hamiltonian) and a population of configurations (hereafter called the old generation). It outputs a new generation that is more optimized than the old one. This function proceeds as follows (see figure 2):

1. If the old generation contains only one configuration, do nothing and return that configuration (the new generation is the same as the old one).

2. Choose $k$ configurations (or parents) at random in the old generation. If possible choose different parents each time.

3. Apply the renormalization procedure to create a renormalized instance together with a set of $k$ renormalized configurations.

4. Apply the local search to these renormalized configurations.

5. Call Next_Generation (recursively) on the renormalized instance and the renormalized configurations. Raise the resulting configurations and apply local search to them. These configurations are the children.

6. Add the children produced to the new generation.

7. Return to step 2 until all the configurations from the old generation have been used as parents at least once.

8. Return the new generation (all the children produced).

The spirit of this function is roughly that each configuration from the old generation gives birth to a more optimized one in the new generation. This new configuration is essentially the old one optimized on many scales by the local search during the recursive calls. In the overall genetic algorithm (see the next section), this function is called repeatedly; thus previous improvements can influence the ones to come.

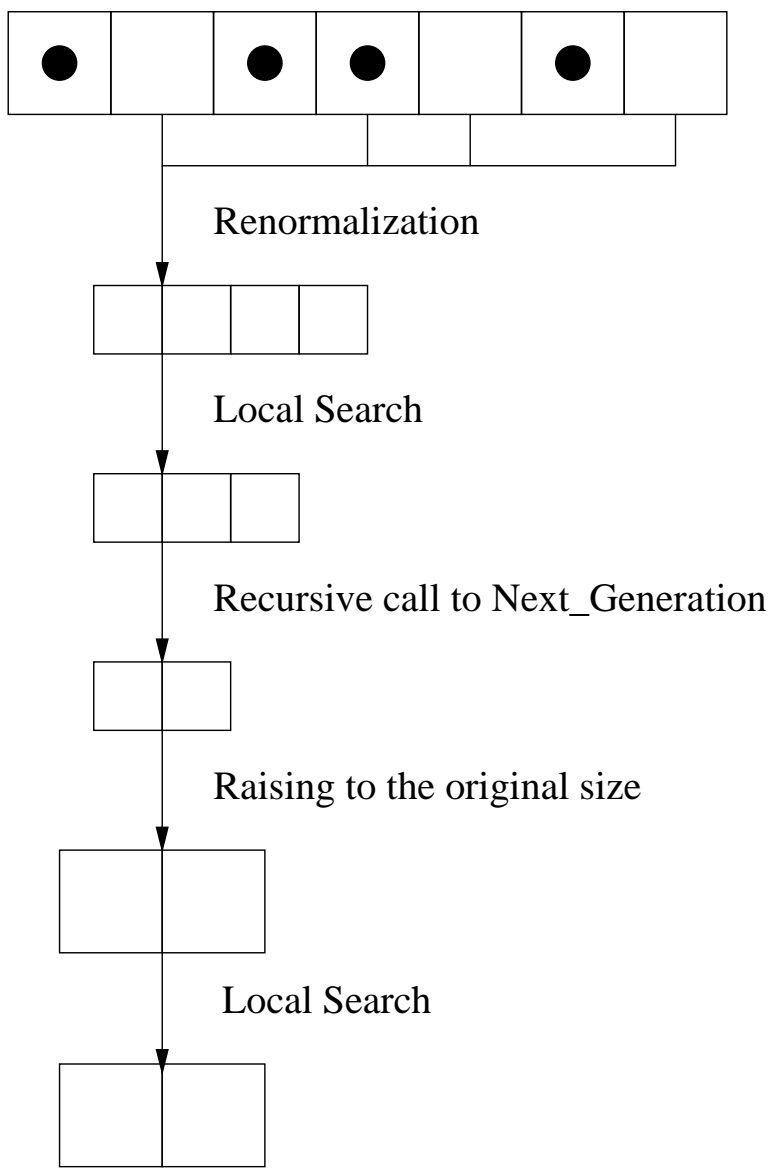

\section{Children produced}

FIG. 2. The inner steps of Next_Generation. The dots mean that the parent has already been used and thus should not be re-used if possible. The steps are repeated until all configurations have been used as parents (i.e., have obtained a dot). 


\section{B. Layout of the algorithm}

At a high level of description, our algorithm computes an optimized configuration (hopefully the ground-state) when it is given an instance. Schematically, it proceeds by following these 5 steps:

1. Randomly generate $M$ configurations.

2. Apply the local search to each one of them.

3. Call the Next_Generation function.

4. Return to step 3 until only one configuration is left.

5. Output this last configuration which is the result.

There is still one detail which must be fixed. We want to be sure that the loop terminates, and thus we want each generation to be strictly more optimized than the previous one. We enforce this at the level of Next_Generation as explained in Appendix 2. Given this last detail, the high-level description of the algorithm is now complete; let us now go on and see how well all this works in practice.

\section{BEHAVIOR OF THE ALGORITHM}

In the rest of this article, we restrict ourselves to Edwards-Anderson (EA) spin-glasses in dimensions 2, 3 , and 4 with periodic boundary conditions and nearest neighbor interactions. Furthermore, our couplings are Gaussian and there is no magnetic field. Thus in equation 1, $h_{i}=0$ and the $J_{i j}$ are independent random variables having a normal distribution of zero mean and variance equal to 1 . (Generally speaking, the algorithm has an easier time finding ground states when there is a magnetic field, justifying our choice of $h_{i}=0$.)

To give the reader some intuition about how the algorithm works, we can follow what happens when going from one generation to the next; that is the object of the next subsection. In the second subsection, we shall see just how powerful the algorithm is by measuring the probability with which it finds the ground state.

\section{A. Qualitative aspects}

In our algorithm, there is only one free parameter: the number $M$ of configurations in the first generation of the genetic algorithm. (The other parameters have been fixed once and for all in the code which thus becomes a "black-box" routine.)

The choice $M=1$ is special as it prevents any renormalization; the algorithm then reduces to applying local search to a randomly generated configuration. How well does that work? Let $\Delta E$ be the difference between the output energy and the ground state energy $E_{0}$. We find empirically that $\Delta E$ is self-averaging as the linear lattice size $L$ goes to $\infty$. Quantitatively, the relative error $\Delta E / E_{0}$ at large $L$ is $5.1 \%$ in $d=2,6.0 \%$ in $d=3$, and $6.2 \%$ in $d=4$. This may seem large but it should be compared to the excesses of over $20 \%$ obtained when using single spin optimization (zero temperature Metropolis).

When $M>1$, the first thing the algorithm does is apply local search to the configurations; thus before calling Next_Generation, we have configurations with the previously given excess energies. Then, as one goes from one generation to the next, the mean energy of the population decreases. This is illustrated in figure 3 for a typical instance with $L=12, d=3$ and $M=1000$. We see that this decrease is initially quite rapid, while at the same time the population size stays fixed at its initial value $M$. For later generations, the mean energy decreases more slowly whereas the population size decreases steadily. Finally, the population size reaches 1 and the algorithm terminates; the last configuration is necessarily the best configuration found throughout the whole run. The pattern shown in the figure is typical and arises for all lattice sizes and dimensions we have investigated.

Naturally, the detailed evolution from generation to generation does fluctuate from instance to instance. Nevertheless, we find that the generation number where the population begins to decrease is quite insensitive to the value of $M$ while it clearly grows for increasing $L$. Furthermore, in the great majority of cases, we find that the final configuration (which is the output) first appears right after the population begins to decrease. Probably the most significant dependence on $M$ concerns the total number of generations produced before termination. The fluctuations in that number are larger than for other observables; also, the trend is towards more generations as $M \rightarrow \infty$. But even there the dependence is not so dramatic; to give some illustrative numbers, consider again $L=12$ for $d=3$. There are typically 10 generations for $M=100$; when $M$ increases, the number of generations increases quite slowly, reaching about 20 for $M=10000$.

\section{B. Probability of finding the ground state}

To a large extent, the power of an optimization algorithm can be quantified by its probability of finding the ground state and by the CPU time required to do so. For a fixed instance and a given number $M$, there is a certain probability $p$ of finding the ground state (remember that our algorithm is stochastic and thus depends on a random number generator). To measure $p$, one should know the ground state, but since that is not the case we proceed self-consistently. We choose a large $M$ and we run the program many times; if the best output (the putative ground state) is found with a high probability (say 90\%) we can reasonably expect that it is the ground state. Morevover we checked the program against a pre- 


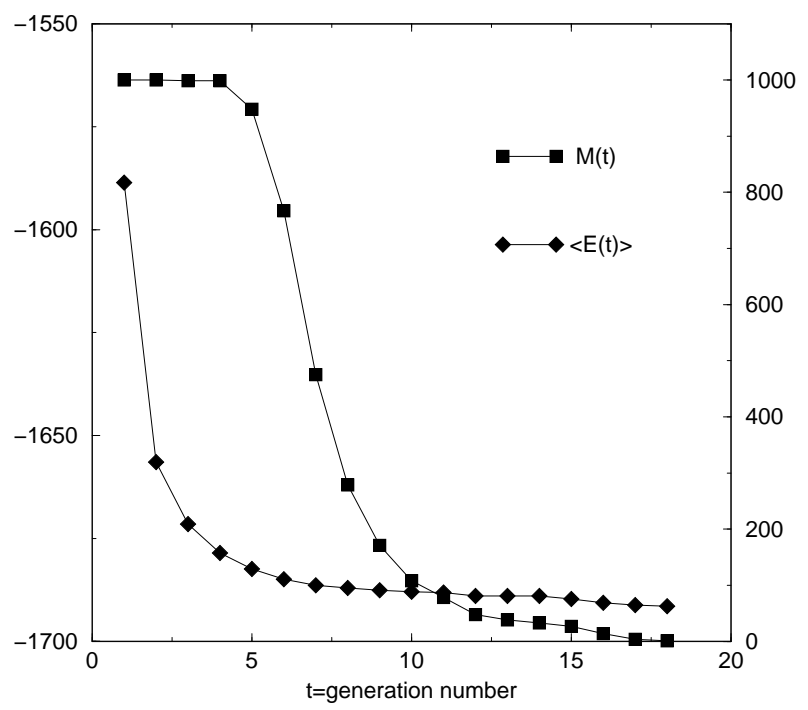

FIG. 3. The evolution with generation number of the mean energy (diamonds and left axis) and of the population size (squares and right axis).

vious version which had been also tested against an exact algorithm 9 .

Once we "know" the ground state, we can measure $p$ for different values of $M$. Empirically we find that for large enough $M$ we have:

$$
p(M) \approx 1-e^{-M / a}
$$

where $a$ is a number which depends on the actual instance. This kind of dependence on $M$ can be motivated by a very simple argument. Let us call $q=1-p$, the probability of not finding the ground state. If we run the program $n$ times (with different random numbers), the probability of not finding the optimum $n$ times is $q^{n}$. If we now run the program with a population of size $n M$ instead of $M$, we should do at least as well and thus $q(n M) \leq q(M)^{n}$. But for large $n M$, the algorithm is expected to do no better, in which case we have the equality $q(n M)=q(M)^{n}$ when $n \rightarrow \infty$; this leads directly to equation 10.

The value of $a$ depends on the instance studied. However, for our benchmarks, we are interested in randomly generated instances of the EA model. In that case, we find that the fluctuations of $a$ decrease as $L$ increases, suggesting that $a$ is self-averaging. Table 1 shows the (mean) values of $a$ obtained from our runs. It is not clear what the function $a(L)$ is, but as a first guess $a$ seems to grow exponentially with $L$ (at least in dimensions 3 and 4). The speed of the program appears to be roughly linear in $N$ and $M$ (for large values). On a $180 \mathrm{MHz} \mathrm{PC}$ running under Linux the coefficient of this law is $1.210^{-4}$ sec. which means that with $M=a$ one requires 24 seconds to find the ground state of a $10^{3} \mathrm{EA}$ spin-glass and 135 seconds for a $12^{3}$ spin-glass.
TABLE I. The value of $a$ (equation 10) for EA spin glasses in dimensions 2,3 and 4 . $L$ is the linear size of the system. The value marked with a (?) are unreliable because it was not clear whether the ground states were found.

\begin{tabular}{||c|c||c|c||c|c||}
\hline \hline \multicolumn{2}{||c||}{ 2D } & \multicolumn{2}{c||}{$3 \mathrm{D}$} & \multicolumn{2}{c||}{$4 \mathrm{D}$} \\
\hline$L$ & $a$ & $L$ & $a$ & $L$ & $a$ \\
\hline 10 & 5.3 & 4 & 4.4 & 3 & 5.6 \\
20 & 9.4 & 6 & 14 & 4 & 27 \\
30 & 11 & 8 & 53 & 5 & 230 \\
40 & 16 & 10 & 200 & 6 & $5500(?)$ \\
60 & 27 & 12 & 650 & & \\
80 & 43 & 14 & $2500(?)$ & & \\
\hline \hline
\end{tabular}

\section{DISCUSSION AND CONCLUSIONS}

As with all known algorithms, it becomes increasingly difficult to find the true ground state as the number of spins increases. What is important though is that larger systems can be tackled 10 with our algorithm than with previous methods11.12. Indeed, before the advent of GRA type algorithms, it was not possible to solve reliably Gaussian EA models of sizes beyond $8 \times 8 \times 8$. (Let us note however that larger sizes cap be tackled for the $\pm J$ model; both Pall3 3 and Hartmann 14 quote results for sizes up to $14 \times 14 \times 14$.) Interestingly, if one looks at currently competitive methods, they all rely on genetic algorithms; it thus seems essential to use multiple configurations (parents) to find very low energy configurations in spin glasses. Our approach takes advantage of this while at the same time allowing optimization on multiple scales; we believe this is the source of our extra performance.

There is every reason to believe that our hierarchical approach can be useful for other optimization problems. Not only is the concept of a block spin natural, but also the use of recursion is not specific to spin glasses. In fact, we have shown previously that a GRA type approach is effective $\mathbb{d}$ for the traveling salesman problem; applications to other problems should appear soon. Naturally, to get the best possible performance, it is necessary to have a good local search method and to define intelligently the renormalization transformation for the problem at hand. A certain amount of problem-specific fine tuning is possible here. In addition, it would be useful to investigate improvements to our GRA that are problem-independent. Among these, we consider particularly promising the possibility of selecting the parents in a non-random fashion in the Next_Generation function, and the maintaining of diversity of the population as the generation number increases.

\section{Acknowledgments}


We thank K. Pal and W. Krauth for helpful comments. J.H. thanks the Max Planck Institute for Polymer Research for its support during this work. The LPTMS is an Unité de Recherche de l'Université Paris XI associée au CNRS.

\section{Appendix 1: Local search}

Our local search algorithm proceeds as follows:

1. Choose a spin that will be the "seed" of the growing cluster. This is done by taking any of the strictly unstable spins of the current configuration; if there are none, choose a spin at random anywhere. Virtually flip this spin and mark it so it cannot be flipped again at any time during the cluster's growth. Compute the new gains of all the other spins of this modified configuration.

2. Add to the cluster the spin with the highest gain, be-it positive or negative with the constraint that the cluster must remain connected. Update the configuration and the gains and again mark this spin so it will not be considered for flipping during the growth of this cluster.

3. Return to step 2 unless there are no more spins to add to the cluster or more than 20 spins have been added to the cluster since the greatest gain has been encountered.

4. If the best cluster encountered during the growth process has a strictly positive gain, flip it.

5. Return to step 1 unless step 4 has already failed 3 times to find a cluster to flip, i.e., all clusters found had non-positive gains.

At the end of this search, all spins have non-positive gains, so we guarantee that the configuration is at least 1-spin-flip optimal. One of the important features of this Kernighan-Lin-like algorithm is that the size of the cluster is not limited (except by $N$ ). It can be very large in practice, especially when the original configuration is random. In order to implement this algorithm efficiently, we use adapted data structures. First, we dynamically maintain a list of the unstable spins; this allows step 1 to be done in time $O(1)$. Second, we use a dynamically maintained heap structure to find the next best spin to add to the cluster; this allows step 2 to be achieved in time $O(\ln K)$ where $K$ is the number of possibilities for the spin to add. In practice the execution time for our implementation is roughly linear in $N$.

\section{Appendix 2: The Next_Generation function}

Here we give a more detailed view of this function. First, consider the choice of $k$, the number of parents used in a renormalization (step 2 of the Next_Generation function). In our approach, $k$ is not a fixed number, it is chosen dynamically for each renormalization in such a way that the renormalized instance is at least $r$ times smaller than the original one (the size of an instance is the number of its spins). In practice, we have set $r=2.5$. We proceed as follows. We first take a "large" number of putative parents (respecting the rule that already used parents should not be used again unless no others are left). For this large value of $k$, the renormalized instance is quite big (the larger $k$, the larger the renormalized instance). Then we decrease $k$ one unit at time until we achieve the wanted size for the renormalized instance (or until we reach $k=2$ ). Naturally, only the $k$ finally selected parents are marked as "used" for the next iteration. It may happen that the renormalized instance is the same as the original one (with very small systems for example). In this case, one possibility is to simply leave the parents unchanged and use them as children (and thus directly go from step 2 to step 6). During the recursive calls the system get smaller and smaller; at some point, the local search is able to find the ground state with high probability, so going to smaller sizes is useless (and uses CPU time). To take advantage of this, we put a barrier at the size $N=15: k$ is no longer decreased if there are 15 or less spins (and if the renormalized instance is smaller than the original one).

A second issue concerns the size of the population returned by Next_Generation: we want to ensure that the number of configurations in the new generation is not greater than in the old one. As described so far, the population size could grow because when producing the last children, parents that have been previously used can be used again (as in figure 2) and thus some parents can produce more than one child. To prevent the population from growing, the simplest method is to remove as many configurations as necessary at step 8 of Next_Generation. We have choosen to do this by removing the worst configurations. Another possibility would be to remove the most similar configurations (but this is computationally more expensive). Thus step 8 is replaced by:

8' Remove the worst configurations from the new generation so it is not larger than the old one. Return the resulting population.

Now the Next_Generation functions properly but we have found it useful to introduce an algorithmic improvement associated with preserving diversity. This is the third point we wish to discuss. In a genetic algorithm, one evolves a population and tries to improve it. One important characteristic is the diversity of the population: the more the configurations differ, the more new configurations one can create. At step 4 of Next_Generation where the local search is applied, something bad can happen. Indeed, since for small enough systems the local search is able to find the ground state with high probability, the (local search) optimization of $k$ different configurations will leave us with just one child, thereby destroying diversity. To prevent this, we have changed step 
5 as follows:

5' If more than one configuration is left after eliminating duplicates in step 4, proceed as in step 5. Else raise this unique configuration and apply local search. The children are the $k$ best configurations out of this one and the $k$ parents.

The last point that needs to be discussed concerns how we force Next_Generation to return a more optimized population than the one in its input, at least when this function is called from the main program. On the contrary, when the function is called from within the recursion, it would be a mistake to impose strict improvement as the population diversity would diminish too quickly. Thus we need to give Next_Generation some additional information. To do that, we add a boolean flag to its arguments which allows one to enforce or not improvement in the new generation. The flag is set to true when initiating the recursion, and its value is passed onward recursively. When the flag value is "false", Next_Generation performs the step 5' described above. When its value is "true", Next_Generation performs instead the original step 5. Furthermore, in the particular case where $k=2$ and the renormalized instance is the same as the original one, instead of returning both parents as children, only the best one is used. Finally, the flag is switched from true to false as soon as step 4 of Next_Generation has improved the configurations, and that new value is passed on recursively.

${ }^{1}$ A. J. Bray and M. A. Moore. Critical behaviour of the three-dimensional Ising spin-glass. Phys. Rev. B, 31:631633, 1985.

2 D. S. Fisher and D. A. Huse. Ordered phase of short-range Ising spin-glasses. Phys. Rev. Lett., 56:1601-1604, 1986.

${ }^{3}$ M.J. Alava, P.M. Duxbury, C. Moukarzel, and H. Rieger. Exact combinatorial algorithms: Ground states of disordered systems. In J. Lebowitz and C. Domb, editors, Phase Transitions and Critical Phenomena. Academic press, 2001.

${ }^{4}$ T. Klotz and S. Kobe. Valley structures in the phase space of a finite $3 \mathrm{~d}$ Ising spin glass with +-I interactions. J. Phys. A, 27:L95, 1994.

${ }^{5}$ S. F. Edwards and P. W. Anderson. Theory of spin-glasses. J. Phys. F, 5:965-974, 1975.

${ }^{6}$ J. Houdayer and O. C. Martin. Renormalization for discrete optimization. Phys. Rev. Lett., 83:1030-1033, 1999. condmat/9901276.

${ }^{7}$ B. Kernighan and S. Lin. An efficient heuristic procedure for partitioning graphs. Bell System Technical Journal, 49:291 - 307, 1970.

${ }^{8}$ N. Kawashima and M. Suzuki. Replica optimization method for ground-state search of random spin systems. J. Phys. A, 25:1055-1070, 1992.
${ }^{9}$ M. Mézard, G. Parisi, and M. A. Virasoro. Spin-Glass Theory and Beyond, volume 9 of Lecture Notes in Physics. World Scientific, Singapore, 1987.

${ }^{10}$ J. Houdayer, F. Krzakala, and O. C. Martin. Large-scale low-energy excitations in 3-d spin glasses. Eur. Phys. J. B., 18:467-477, 2000. cond-mat/0009382.

${ }^{11}$ K. F. Pal. The ground state of the cubic spin glass with short-range interactions of Gaussian distribution. Physica A, 233:60-66, 1996.

${ }^{12}$ M. Palassini and A. P. Young. Nature of the spin glass state. Phys. Rev. Lett., 85:3017, 2000. cond-mat/0002134.

${ }^{13}$ K. F. Pal. The ground state energy of the EdwardsAnderson Ising spin glass with a hybrid genetic algorithm. Physica A, 223:283-292, 1996.

${ }^{14}$ A. Hartmann. Scaling of stiffness energy for 3D +/-J Ising spin glasses. Phys. Rev. E, 59:84-87, 1999. 